\title{
Intracranial stent placement for an acute ischemic stroke in a young patient with intracranial arterial dissection
}

\begin{abstract}
A 36-year-old Caucasian woman with a history of bipolar disorder was brought to the hospital after she was found down on floor. On examination, there was left hemiparesis, forced right gaze deviation and severe dysarthria. She was last seen normal six hours prior to the arrival and a computerized tomography (CT) of the head was unremarkable, except for a hyperdense right middle cerebral artery (MCA) sign. Subsequent magnetic resonance imaging (MRI) of the brain revealed early ischemic changes in the right hemisphere, less than one third of the right middle cerebral artery vascular territory. An MRI angiography showed a stump in the right M1 segment of the MCA. Vessel wall imaging MRI showed an intramural hematoma in the right M1 segment. The diagnosis of right M1 occlusion due to intracranial dissection was made and intracranial stent was planned, performed. She had an excellent clinical and radiological outcome on follow up.
\end{abstract}

Keywords: middle cerebral artery, dissection, intracranial stent, ischemic stroke
Volume 9 Issue 4 - 2019

Pranitha Reddy Arrabyru, Rakesh Khatri, Alberto Maud, Gustavo Jose Rodriguez Neurology Department, Texas Tech University Health Sciences Center, USA

Correspondence: Pranitha Reddy Arrabyru, Neurology Department, Texas Tech University Health Sciences Center, El Paso,Texas, USA, Email arrabairupranithareddy@gmail.com

Received: June 20, 2019 | Published: July 09, 2019
Abbreviations: MRI, magnetic resonance imaging; MCA, middle cerebral artery; LVO, large vessel occlusion; ICD, intracranial arterial dissection; CAD, cervical arterial dissection; DSA, digital subtraction angiography; NIHSS, national institutes of health stroke scale

\section{Introduction}

Given the results of the recent acute ischemic stroke trials, mechanical thrombectomy is considered the standard of care for acute ischemic stroke related to a large vessel occlusion (LVO) within 6hours in the anterior circulation. ${ }^{1}$ Treatment can also be performed between 6 and 24hours when there is evidence of salvageable tissue. ${ }^{1,2}$ A meta-analysis of the five successful randomized controlled trials, revealed that mechanical thrombectomy using a stentriever fails recanalization in approximately $30 \%$ of all cases. ${ }^{3}$ Anatomical challenges (as in severe arterial tortuosity), tandem occlusions, large thrombi in the internal carotid artery, physical properties of the thrombi (soft/hard/organized), and underlying intracranial arterial disease like in atherosclerosis or dissection are possible aetiologies for the refractoriness. ${ }^{4}$

Arterial dissection is the most common cause of stroke in the young. ${ }^{5-7}$ Intracranial arterial (ICD) dissection particularly affecting the middle cerebral artery is a rare cause of ischemic stroke, ${ }^{8}$ compared to the cervical arterial dissection (CAD). Although digital subtraction angiography (DSA) is considered the gold standard diagnostic imaging, the typical findings of double lumen or intimal flap are seen in about $10 \%$ of the cases. ${ }^{9}$ The vessel wall imaging MRI (VW MRI) is emerging as a diagnostic tool to diagnose dissection, although at times it is difficult to differentiate between an intramural hematoma and an intraplaque hemorrhage. ${ }^{10}$ We present a case of an acute intracranial dissection that was successfully treated with primary stent placement.

\section{Case presentation and observation}

A 36-year-old Caucasian woman presented to the Emergency Department after she was found down on floor, at her home. She had a history of bipolar disorder, no head trauma or vascular risk factors. She was last seen normal about six hours prior to her arrival to the hospital. Upon neurological examination, she was noted to have dense left hemiparesis, forced right gaze deviation and severe dysarthria, the National Institutes of Health Stroke Scale (NIHSS) was 10. A computerized tomography of the head demonstrated a hyperdense right middle cerebral artery (MCA) and no other obvious hyperdensities associated. An emergent magnetic resonance imaging (MRI) of the brain was performed (Figure 1). The MRI of the brain demonstrated early ischemic changes in the right hemisphere, less than one third of the right middle cerebral artery vascular territory, the MRI angiography showed an occlusion of the right M1 segment and an intramural hematoma was also noted in the right M1 segment. Knowing this information, the patient was emergently taken to the angio suite and the diagnosis was made of right M1 occlusion due to an intracranial dissection (Figures 2A-2C).

A 0.014-inch Synchro-2 standard microwire (Stryker Corporation, Kalamazoo, Michigan, USA) was used to reach (was advanced towards) the lesion and a Prowler Select Plus microcatheter (Codman, Raynham, Massachusetts, USA) was advanced over this guide wire. The wire was then removed and a double angiogram (via simultaneous microcatheter and guide catheter injection) demonstrated the area of stenosis/occlusion. An Enterprise 4.5x22mm (Codman, Raynham, MA) stent was then placed across the area of stenosis/occlusion. The patient was given an intravenous load of Integrilin (Eptifibatide) $135 \mathrm{mcg} / \mathrm{Kg}$ and was loaded orally with aspirin $325 \mathrm{mg}$ and clopidogrel $300 \mathrm{mg}$, to follow with daily aspirin $81 \mathrm{mg}$ and clopidogrel $75 \mathrm{mg}$. 


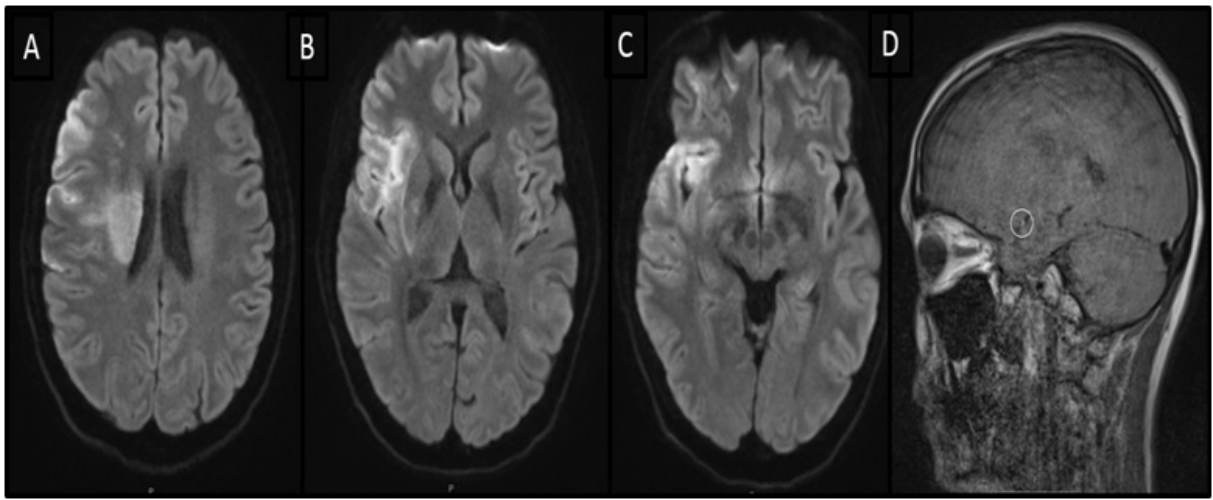

Figure I Magnetic resonance imaging (MRI). (A-C) Diffusion weighted imaging (DWI), axial view demonstrating early ischemic changes in the right hemisphere. (D) Vessel wall MRI, sagittal view showing an intramural hematoma in MI segment of the right middle cerebral artery (MCA).
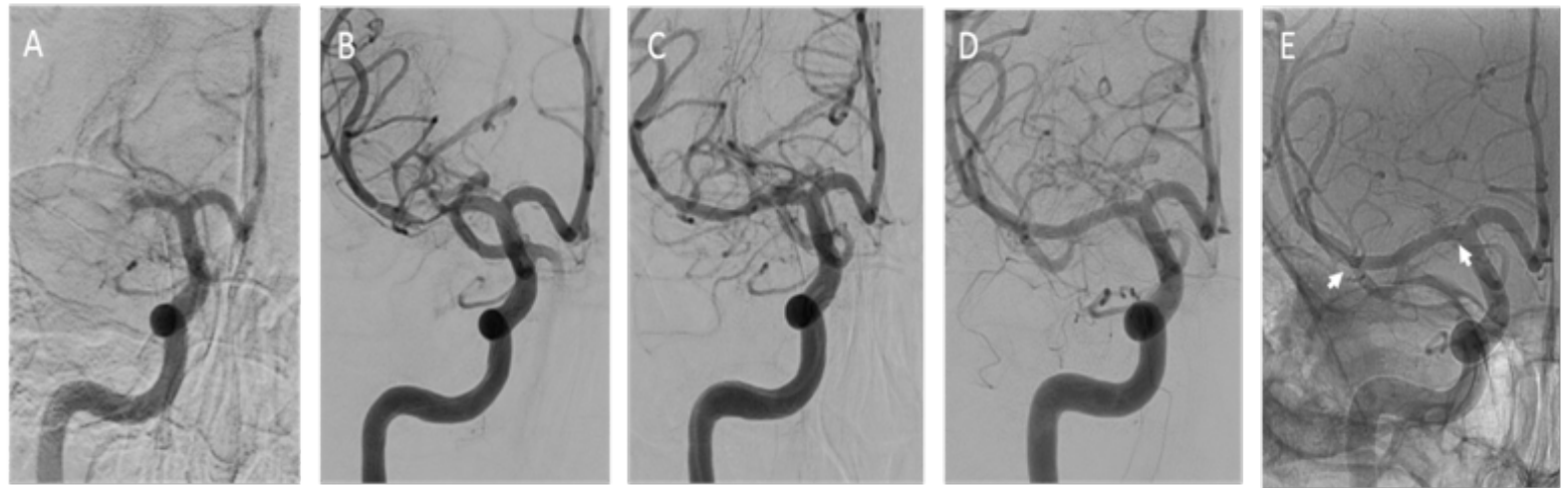

Figure 2 Digital subtraction imaging. (A) Anterior posterior view contrast angiogram demonstrating occlusion of the right MI segment. (B) Double angiogram demonstrating the disrupted vessel. (C) Angiogram showing stent placement. (D) Follow up at 6 months. (E) Unsubtracted angiography demonstrating the stent at six months (arrows).

Clinically the patient improved on the table and was able to raise the left hemibody against gravity (MRC grading for muscle strength=4), we noticed complete resolution of the gaze deviation and improved dysarthria. A follow up CT head was performed on the day 1 (Figure 3). The patient was discharged home with outpatient rehabilitation and NIHSS $=2$.

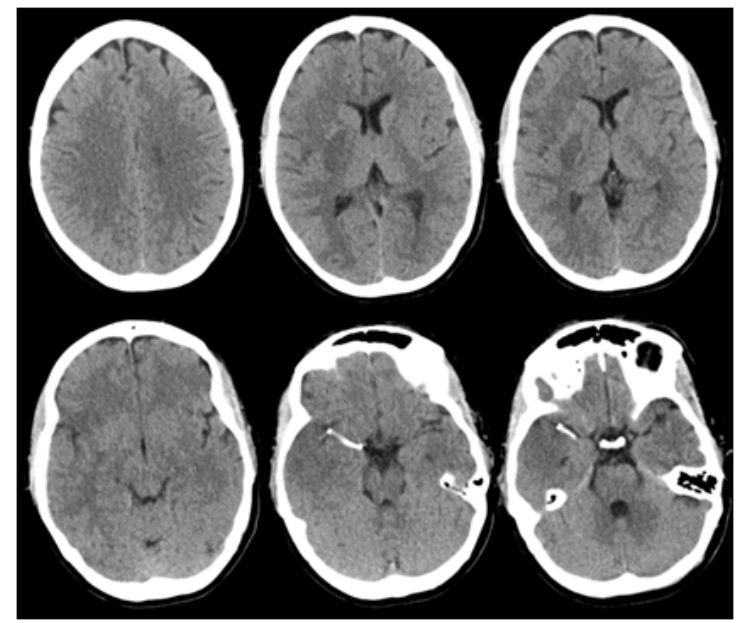

Figure 3 Computerized tomography (CT) head demonstrating intracranial stent in the right MI segment of the middle cerebral artery, on day I.

At three months, the patient had returned to her baseline with a modified Rankin score (mRS) of 0 and NIHSS of 0 . Despite the lack of symptoms, the dual antiplatelet therapy was continued for about 6 months, time when she had a follow up angiogram (Figure 2D\&2E).

\section{Discussion}

We here present a successful case of an acute ischemic stroke due to an intracranial dissection treated with primary stent placement. Isolated middle cerebral arterial dissection (MCAD) is a poorly understood cause of stroke. The incidence is still not known. ${ }^{8}$ It should be considered when a young patient has a middle cerebral artery (MCA) territory infarct with stenosis/occlusion at the proximal segment of the MCA. ${ }^{11}$ The M1 segment of the MCA is most commonly involved in MCAD due to its proximity to the sphenoid wing. ${ }^{12,13}$ It is more commonly reported in Asians, and in the absence of obvious trauma, the etiology of most of the cases is spontaneous/idiopathic. ${ }^{7,12}$ Risk factors include history of trivial trauma, migraine, connective tissue disorders, and vascular risk factors like hypertension, hyperlipidemia, tobacco abuse, diabetes mellitus, and oral contraceptives. ${ }^{8,12}$

Isolated MCAD is more likely to result in ischemic stroke rather than hemorrhagic stroke syndromes. ${ }^{12}$ The proposed mechanism comprises the hemodynamic disturbance resulting from the stenosis or occlusion due to the arterial wall hematoma and/or the distal embolism of thrombi. ${ }^{6,8}$ The presenting symptoms of MCAD are variable, and the most common are headache, seizure, nausea, vomiting, tinnitus, and neurological deficits depending on the portion of middle cerebral artery involved.(12) Approximately $20 \%$ can present with no neurological deficits. ${ }^{8}$ Jisang Park et al. reported a proximal M1 portion of the right MCA dissection with active inflammation in a 33 year old Asian man 
who presented with recurrent left sided hemidystonia with smoking as the only stroke risk factor. ${ }^{14}$ The patient in this case was noted to have dense left hemiparesis, forced gaze deviation, and severe dysarthria due an occlusion of right M1 segment of MCA.

The diagnosis of MCAD depends on both invasive and noninvasive methods. The modalities commonly used include digital subtraction angiography (DSA), computed tomography/angiography (CT/CTA), magnetic resonance imaging/ angiography (MRI/ MRA), clinical and pathological evidence/ autopsy, transcranial Doppler (TCD). ${ }^{12}$ Every imaging technique has its own limitation. Although DSA is traditionally considered the gold standard for detecting dissections, there is no common agreement as to which diagnostic tool is most useful. The identification of the subintimal dissection is easier to diagnose with DSA, however the subadventitial dissection forming a pseudoaneurysm without narrowing the arterial lumen and flow impairment can be missed. ${ }^{10}$ Vessel wall magnetic resonance imaging (VW-MRI) appears to be superior. ${ }^{10}$ In addition in the presence of a complete vessel occlusion, the DSA has poor diagnostic value for dissection. In our case, the MRI of the brain demonstrated early ischemic changes in the right hemisphere. The VW-MRI showed an intramural hematoma in the right M1 segment of the middle cerebral artery, leading to the diagnosis.

The best therapeutic intervention for isolated MCAD is still unclear. ${ }^{8}$ The treatment strategies in the literature include the early use of antiplatelet agents, anticoagulation, conservative management,

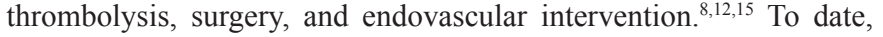
no study has been performed to address the safety and efficacy of the different therapeutic alternatives. Some authors recommend against the use of anticoagulants as they consider those may promote progression of dissection and the intramural hematoma while others prefer anticoagulants as they slow down the progression of thrombosis. ${ }^{12}$ The safety of intravenous thrombolysis for intracranial artery dissection is unclear. ${ }^{12}$ Patients who are not responding to medical management, intracranial stent placement can be considered. ${ }^{6}$ Few cases are available in the literature about acute ischemic stroke in the setting of intracranial dissection. In Kim's et al. study, in which a series of cases with intracranial dissection were studied, two patients presented with acute symptoms and had a self-expanding stent placed for acute symptomatic M1 dissection and internal carotid and M1 dissection, both experienced clinical improvement of the dissectionrelated stenosis, and mRS at 3months of 2 and 1, NIHSS of 7 and 0 at discharge. ${ }^{6}$

It is possible that intracranial dissection is the main cause of mechanical thrombectomy failure in young patients with stroke. As repeated passes of a stent retriever in the disrupted vessel may worsen the dissection and less likely to recanalize, early suspicion and recognition of intracranial dissection can lead the operator about a different approach, successfully as in our case.

\section{Conclusion}

i. Presenting symptoms of MCAD are variable, idiopathic etiology is common.

ii. Consider MCAD as the diagnosis when a young patient has a MCA territory infarct with stenosis/occlusion of the M1 segment.

iii. Consider intracranial stent placement as a safe and effective therapeutic intervention in young patients with suspected intracranial arterial dissection as mechanical thrombectomy may fail.

\section{Conflicts of interests}

The authors declare no conflicts of interests

\section{Authors' contributions}

Pranitha Reddy Arrabyru involved in manuscript writing; Rakesh Khatri, Alberto Maud involved in critical revision of the manuscript; Gustavo Jose Rodriguez involved in critical revision of the manuscript and also involved in patient care. All the authors have read and agreed to the final manuscript.

\section{References}

1. Powers WJ, Rabinstein AA, Ackerson T, et al. Guidelines for the Early Management of Patients With Acute Ischemic Stroke: A Guideline for Healthcare Professionals From the American Heart Association/ American Stroke Association. Stroke. 2018;49(3):e46-e110.

2. Nogueira RG, Jadhav AP, Haussen DC, et al. Thrombectomy 6 to 24 Hours after Stroke with a Mismatch between Deficit and Infarct. $N$ Engl J Med. 2018;378(1):11-21.

3. Goyal M, Menon BK, van Zwam WH, et al. Endovascular thrombectomy after large-vessel ischaemic stroke: a meta-analysis of individual patient data from five randomised trials. Lancet. 2016;387(10029):1723-1731.

4. Kim BM. Causes and Solutions of Endovascular Treatment Failure. $J$ Stroke. 2017;19(2):131-142.

5. Schievink WI. Spontaneous dissection of the carotid and vertebral arteries. N Engl J Med. 2001;344(12):898-906.

6. Kim DJ, Kim BM, Suh SH, et al. Self-expanding stent placement for anterior circulation intracranial artery dissection presenting with ischemic symptoms. Neurosurgery. 2015;76(2):158-164.

7. Choi YJ, Jung SC, Lee DH. Vessel Wall Imaging of the Intracranial and Cervical Carotid Arteries. J Stroke. 2015;17(3):238-255.

8. Zhang L, Nan G, Mao Y, et al. Rapid improvement of angiostenosis due to isolated middle cerebral artery dissection: A case report. Medicine (Baltimore). 2018;97(4):e9695.

9. Siddiq F, Chaudhry SA, Das P, et al. Occurrence and prognostic significance of cervical pseudodissection phenomenon associated with acute intracranial internal carotid artery occlusion. $J$ Neuroimaging. 2013;23(3):384-390.

10. Tan HW, Chen X, Maingard J, et al. Intracranial Vessel Wall Imaging with Magnetic Resonance Imaging: Current Techniques and Applications. World Neurosurg. 2018;112:186-98.

11. Lee JS, Bang OY, Lee PH, et al. Two cases of spontaneous middle cerebral arterial dissection causing ischemic stroke. J Neurol Sci. 2006;250(1-2):162-166.

12. Asaithambi G, Saravanapavan P, Rastogi V, et al. Isolated middle cerebral artery dissection: a systematic review. Int J Emerg Med. 2014;7(1):44.

13. Mobbs RJ, Chandran KN. Traumatic middle cerebral artery occlusion: case report and review of pathogenesis. Neurol India. 2001;49(2):158 161.

14. Park J, Kwon KY. Recurrent episodes of isolated hemidystonia in a young adult: Dissection-associated stenosis in the middle cerebral artery. Parkinsonism Relat Disord. 2016;30:86-88.

15. Lin CH, Jeng JS, Yip PK. Middle cerebral artery dissections: differences between isolated and extended dissections of internal carotid artery. $J$ Neurol Sci. 2005;235(1-2):37-44. 